\title{
Cerebrospinal Fluid Concentrations of Corticotropin-Releasing Hormone (CRH) and Diazepam-Binding Inhibitor (DBI) during Alcohol Withdrawal and Abstinence
}

\author{
Bryon Adinoff, M.D., Ray Anton, M.D., Markku Linnoila, M.D., Ph.D., Alessandra Guidotti, Ph.D., \\ Charles B. Nemeroff, M.D., Ph.D., and Garth Bissette, Ph.D.
}

The neuropeptides diazepam binding inhibitor (DBI) and corticotropin-releasing hormone (CRH) elicit anxietylike symptonts alhen administered intracerebroventricularly to laboratory animals. Because of the similarities between the symptoms of certain anxiety states and the alcohol withdrawnl syndrome, we hypothesized that increased secretion of either of these endogenous neuropeptides may, at least in part, be responsible for the symptoms of alcolol withdrawal. We therefore measured $D B I$ and $C R H$ concentrations in cerebrospinal fluid (CSF) of 15 alcoholdependent patients during acute withdrawal (Day 1) and again at 3 aveek's abstinence (Day 21). In addition, plasma concentrations of cortisol were measured to eraluate the

KEY WORDS: Alcohol inithtrainal; Alcoholism; Diazepam binding inhibitor; Corticotropin releasing hormone; GABA receptor; HPA axis

From the Department of P'sychiatry (BA), University of Texas Southwestern Medical Center at Dallas and VA Medical Center, Dallas, TX; the Department of Psychiatry (RA). Medical University of South Carolina, Charleston; the Laboratory of Clinical Studies (ML), DICBR, National Institute on Alcohol Abuse and Alcoholism, Bethesda, MD; the Center for Neuropharmacology (AG), The Nathan S. Kline Institute, Orangeburg, NY; the Department of Psychiatry and Behavioral Sciences (CBN), Emory University School of Medicine, Atlanta, GA; and the Department of Psychiatry (CBB), Lniversity of Mississippi, Jackson, MS.

Address correspondence to: Dr. Bryon Adinoff, 116A, VA Medical Center, 4500 S. Lancaster Road, Dallas, TX, 75216.

Received April 19, 1995; rerised August 29, 1995; accepted October 17,1995 . relationship between pituitary-adrenal axis activation and CSF CRH concentrations. CSF CRH $(\mathrm{p}<.04)$, but not CSF DBI, was significantly higher on Day 1 than on Day 21. Although there was a significant decrease in plasma cortisol from Day 1 to Day $21(\mathrm{p}<.001)$, a significant correlation between CSF CRH and plasma cortisol concentrations was not observed at either time point. Neither CSF neuropeptide correlated with clinical measures of zoithdrawal severity. These tentative findings may implicate $C R H$, but not DBI, in the pathogenesis of alcohol a'ithdrazial. Alternately, the central release of $\mathrm{CRH}$ and DBI may not be adequately reflected in lumbar CSF.

[Neuropsychopharmacology 15:288-295, 1996]

The clinical and neurobiological alterations that occur during the alcohol withdrawal syndrome bear remarkable similarities to those of anxiety attacks. Symptomatically, to differentiate between acute alcohol withdrawal and either acute or chronic anxiety syndromes can be difficult for both clinicians and patients alike (see review in Kushner et al. 1990). George et al. (1988), for example, has reported that alcohol-dependent patients with coexisting panic disorder are not able to differentiate the signs and symptoms of alcohol withdrawal from those of panic (only tremor was reported as being significantly more severe during alcohol withdrawal than during panic attacks). The subjective sense of anxiety, as well as physiological changes of tremor, increased heart rate and blood pressure, and diaphoresis are observed in both syndromes. 
Biological systems involved in both alcohol withdrawal and anxiety include the $\mathrm{GABA}_{\mathrm{A}}$-benzodiazepine (BZ) receptor complex and the hypothalamic-pituitary-adrenal (HPA) axis. Pharmacological studies have suggested that both anxiety (Breier and Paul 1990) and alcohol withdrawal (Morrow et al. 1988) are associated with an attenuation in chloride flux through the $\mathrm{GABA}_{\mathrm{A}}-\mathrm{BZ}$ receptor complex ion-gated channel. Pituitary-adrenal axis activation, induced by the release of hypothalamic corticotropin-releasing hormone (CRH), is also observed during both acute anxiety states and alcohol withdrawal (Mendelson and Stein 1966; Adinoff et al. 1991a). Furthermore, both the BZ receptor and the HPA axis can be affected by endogenous compounds known to elicit anxietylike symptoms.

Diazepam binding inhibitor (DBI) binds at the $\mathrm{BZ}$ receptor site and acts as a negative allosteric modulator of the BZ receptor (see review in Costa and Guidotti 1991). DBI thus elicits an effect opposite to that of the BZs, producing a proconflict (anxiogenic) response that can be antagonized by the $\mathrm{BZ}$ receptor antagonist flumazenil (Ferrero et al. 1986). DBI is found in many cortical and subcortical brain areas, including the hypothalamus, amygdala, cerebellum, thalamus, and hippocampus, and can be measured in the cerebrospinal fluid (CSF). Elevated levels of a DBI-like immunoreactive compound has been reported in patients diagnosed with depression with (Barbaccia et al. 1986) and without (Roy 1991) concomitant anxiety. DBI concentrations in the hippocampus, an area central to the regulation of affect, are also increased in rats following acute noiseinduced stress (Ferrarese et al. 1991). Miyata et al. (1987) have reported that both the expression of DBI mRNA and DBI concentrations are higher in the cerebellum and cortex of rats administered diazepam over 10 to 15 days, perhaps as a compensatory response to the positive allosteric modulating effects of diazepam. Because the chronic administration of diazepam and alcohol both induce similar augmentation in BZ receptor chloride flux (Sudzak et al. 1986), a compensatory increase in CNS concentrations of DBI might also be predicted following the chronic ingestion of alcohol.

Hypothalamic CRH activates the pituitary-adrenal axis in response to a variety of stressors. In addition, $\mathrm{CRH}$ is heterogenously distributed throughout the CNS, including the cerebral cortex and limbic system (Millan et al. 1986), and when injected intracerebroventricularly ( $\mathrm{CCV}), \mathrm{CRH}$ coordinates the immune, behavioral, and autonomic responses to stress (Sutton et al. 1982; Rock et al. 1984; Britton et al. 1986). In addition to its endocrine role, the ICV administration of CRH produces behavioral effects that are similar to the behaviors observed in animal models of stress. Transgenic mice exhibiting CRH overproduction display increased anxiogenic behavior; this behavior is reversed following the ICV administration of a CRH-receptor antago- nist (Stenzel-Poore et al. 1994). Similarly, stress-induced behaviors in rats are antagonized by the administration of the CRH-receptor antagonist, $\mathrm{CRH}_{9-41}$, into the central amygdala (Swiergiel et al. 1993). In addition, ICV administered CRH has been shown to stimulate the release of norepinephrine from the locus corruleus (Valentino and Foote 1986). The relationship of CRH to both HPA axis functioning and stress-related behaviors, coupled with the development of sensitive and specific $\mathrm{CRH}$ radioimmunoassays, has served as an impetus to measure $\mathrm{CRH}$ concentrations in several psychiatric disorders. CSF CRH concentrations have been reported to be increased in patients with depression (Nemeroff et al. 1984; Banki et al. 1987) and anorexia nervosa (Kaye et al. 1987), although both normal (Kling et al., 1991; Roy et al. 1987) and decreased (Geracioti et al. 1992) concentrations of CSF CRH have been observed in depressed patients. However, it is not clear whether alterations in CSF CRH concentrations are related primarily to disturbances in HPA axis functioning per se or to changes in CRH release from other CNS CRH-containing neurons.

In summary, (1) the ICV administration of both DBI and $\mathrm{CRH}$ induce behavioral changes similar to the signs and symptoms of alcohol withdrawal, and (2) similar alterations in DBI- and CRH-related biological systems are observed in both anxietylike states and alcohol withdrawal. Thus, we sought to determine whether changes in CSF DBI and CRH concentrations occur during alcohol withdrawal in human subjects and whether the altered secretion of either of these compounds is related to the signs and symptoms of alcohol withdrawal. In addition, the alcohol withdrawal syndrome offers a naturalistic model of a severe stress response with a relatively predictable time course of induction and recovery, providing a unique opportunity to study alterations in these stress-related peptides in a period of rapid physiological change. The relationship of these two putative neurotransmitters to each other was of particular interest because of the reported positive correlation between CSF DBI and CRH in both healthy controls and patient groups (Roy et al. 1990), suggesting a physiological interaction between these two neuropeptides. In this study, we therefore measured CSF concentrations of DBI and CRH in alcohol-dependent patients during acute alcohol withdrawal and at 3 weeks' abstinence. This work has previously been presented (Adinoff et al. 1992).

\section{METHODS}

\section{Patients}

Fifteen alcohol-dependent men (age mean \pm SD, $43.5 \pm$ 9.1 years) were studied at the Veterans Affairs Medical Center in Charleston, SC. These patients were screened from over 700 inpatient admissions to the substance 
abuse unit over a 2-year period. Diagnosis of alcohol dependence was made according to DSM-III-R criteria. Patients must have been drinking at least six standard drinks daily for at least 2 weeks prior to admission and have ingested alcohol within 24 hours prior to admission to the study. Drinking history was obtained from the patient and confirmed by family or friends, when present, accompanying the patient. Patients with concurrent use of other psychoactive substances (except marijuana) within the previous 14 days or with a history of other psychoactive substance dependence within the previous 30 days were excluded from the study. The absence of other substance use was substantiated by urine drug screen. Psychiatric exclusions included a past or present diagnosis of schizophrenia or bipolar affective disorder. These exclusions were based on clinical interview with the patient and accompanying family or friends at the time of admission as well as a review of previous hospital records, when available. All patients with a history of seizures were excluded. Other exclusion criteria included present use of medications (i.e., all psychotrophics, calcium-channel blockers, betablockers, hypoglycemics, anticonvulsants, sympathomimetics) or medical conditions (including cirrhotic liver disease) that might significantly interfere with the course of alcohol withdrawal, CABA-ergic system, or HPA axis functioning.

\section{Procedure}

Patients were enrolled into the study on the day of admission to the hospital (Day 1). After obtaining informed consent, patients were placed at strict bedrest and an intravenous catheter was inserted into the forearm. Patients remained at bed rest until the lumbar puncture was completed, and meals were offered at routine meal times. Alcohol withdrawal severity was assessed with the Clinical Instrument Withdrawal Assessment for Alcohol-Revised (CIWA-AR) (Sullivan et al. 1989). The CIWA-AR is a standardized, observer administered rating scale allowing for the rapid, objective determination of withdrawal severity. Because this measure was obtained on an hourly basis, the "insomnia" score was not obtained. In the experience of these investigators, subjects scoring above 9 exhibit at least moderate symptoms of alcohol withdrawal.

The study was initiated when the breath alcohol concentration (obtained by Alco-Sensor II breathalyzer) was less than $20 \mathrm{mg} \%$ and withdrawal severity, as assessed with the CIWA-AR, was 10 or greater. The lumbar puncture was performed when the CIWA-AR was rated at 10 or above $(n=9)$. If the CIWA-AR did not reach this level of severity by the morning of the second day of withdrawal, a lumbar puncture was performed at this time $(n=6)$. Three weeks after the first lumbar puncture (Day 21), the same patients underwent a second lumbar puncture. Lumbar punctures on Day 21 were performed at the same time of day as the procedure on Day 1 to adjust for diurnal changes. Intravenous catheters were inserted at least 90 minutes prior to the second lumbar puncture.

Before each lumbar puncture, measures of withdrawal severity (CIWA-AR), anxiety (Speilberger State Anxiety Inventory and Hamilton Rating Scale for Anxiety), depression (Zung Self-Rating Depression Scale), blood pressure (manually with sphygmomanometer), and heart rate (by cardiac Vagal Tone Monitor or palpation) were obtained. Plasma was obtained for cortisol concentrations.

\section{Bioassays}

CSF CRH and DBI was determined as previously described by Bisette et al. (1985) and Barbaccia et al. (1986), respectively. Intra-assay variance of CRH was less than $6 \%$; sensitivity was 0.625 pg per tube. Intra-assay variance of DBI was less than $5 \%$. Cortisol was measured by fluorescence polarization immunoassay.

\section{Statistics}

Group differences between Day 1 and Day 21 were assessed statistically with paired Student's t-test. Our hypothesis was that both DBI and CRH would be higher on Day 1 than on Day 21. Therefore, a one-tailed $t$-test was used to assess statistical significance. Correlations between individual variables were examined with Pearson's $r$.

\section{RESULTS}

Patients reported a problematic drinking history of $24.7 \pm$ 6.9 years (mean \pm SD) and a daily intake of $378.58 \pm$ $154.13 \mathrm{~g} /$ day ethanol in the 2 weeks before admission. CIWA-AR scores at the time of the lumbar puncture on Day 1 ranged from 2 to $19(12.0 \pm 5.9)$. Measures of withdrawal severity, depression, anxiety, pulse, and blood pressure were all significantly higher on Day 1 than Day 21 (see Table 1). Plasma concentrations of cortisol were also markedly higher on the first day of withdrawal than on Day 21 of abstinence $(p=.001)$.

$\mathrm{CRH}$ concentrations were significantly higher on Day 1 than Day 21 ( $p=.037$ ) (see Table 1 and Figure 1). We further examined changes in $\mathrm{CRH}$ that were greater than $12 \%$ (twice the intra-assay variance) and $1.25 \mathrm{pg}$ 
Table 1. Clinical and Biological Measures during Acute Withdrawal (Day1) and Following 3 Weeks' Abstinence (Day 21) in 15 Patients

\begin{tabular}{lcccc} 
& Day 1 & Day 21 & $t$ & $\begin{array}{c}p \\
\text { Value }\end{array}$ \\
\hline CIWA-AR & $12.0=5.9$ & $2.6 \pm 3.7$ & 6.37 & 0.0001 \\
Speilberger state & $54.0 \pm 13.5$ & $35.5 \pm 10.0$ & 4.83 & 0.0002 \\
Z.ung depression & $46.0 \pm 12.1$ & $36.7 \pm 9.5$ & 3.94 & 0.0008 \\
Hamilton anxiety & $20.6 \pm 7.8$ & $6.2 \pm 6.1$ & 6.92 & 0.0001 \\
Systolic BP $(\mathrm{mmH})$ & $142.1 \pm 16.3$ & $122.2 \pm 11.0$ & 3.92 & 0.001 \\
Heart rate $(\mathrm{bpm})$ & $75.1 \pm 10.4$ & $68.9 \pm 8.8$ & 2.10 & 0.027 \\
Cortisol & $13.0 \pm 7.3$ & $5.6 \pm 4.2$ & 3.77 & 0.001 \\
DBl $(\mathrm{pg} / \mathrm{ml})(\mathrm{n}=14)$ & $19.3 \pm 11.5$ & $15.6 \pm 4.6$ & 1.16 & 0.174 \\
CRH $(\mathrm{pg} / \mathrm{ml})$ & $45.0=20.1$ & $39.6 \pm 13.5$ & 1.92 & 0.037 \\
\hline
\end{tabular}

Analysis are one-tailed t-te'sts

(twice the assay sensitivity) between the two study days. Using these criteria, six of the 15 patients had CSF $\mathrm{CRH}$ concentrations on Day 1 greater than on Day 21; two patients were lower on Day 1 than Day 21. CSF concentrations of DBI were not significantly different on Day 1 from those on Day 21.

There was a high correlation between CSF DBI and CRH on Day $21(r=.71, \mathrm{df}=14, p=.004)$. A significant correlation between the two neuropeptides was not observed on Day $1(r=0.20, \mathrm{~d} f=14, p=.40)$. However, following the exclusion of a patient with CSF DBI concentration of $56.2 \mathrm{pg} / \mathrm{ml}$ (greater than six standard deviations above the mean DBI concentration on Day 1) from the analysis, a significant correlation $(r=.75, \mathrm{df}=$
$13, p=.01$ ) between CSF DBI and CRH on Day 1 was evident. Significant correlations between the clinical variables (CIWA-AR, Speilberger, Ham-A, and Zung ratings and blood pressure and heart rate) and the concentrations of the neuropeptides DBI and CRH were not observed on either Day 1 or Day 21. Similarly, plasma cortisol did not significantly correlate on either study day with DBI (Day 1: $r=.012, \mathrm{df}=14$; Day 21: $r=$ $-0.515, \mathrm{df}=14$ ) or CRH (Day 1: $r=-0.382, \mathrm{df}=15$; Day 21: $r=-0.489, \mathrm{df}=15$ ). Change scores (Day $1-$ Day 21) between clinical variables and plasma cortisol concentrations did not significantly correlate with change scores of CSF DBI or CRH concentrations, except for an unexpected negative correlation between CSF CRH and

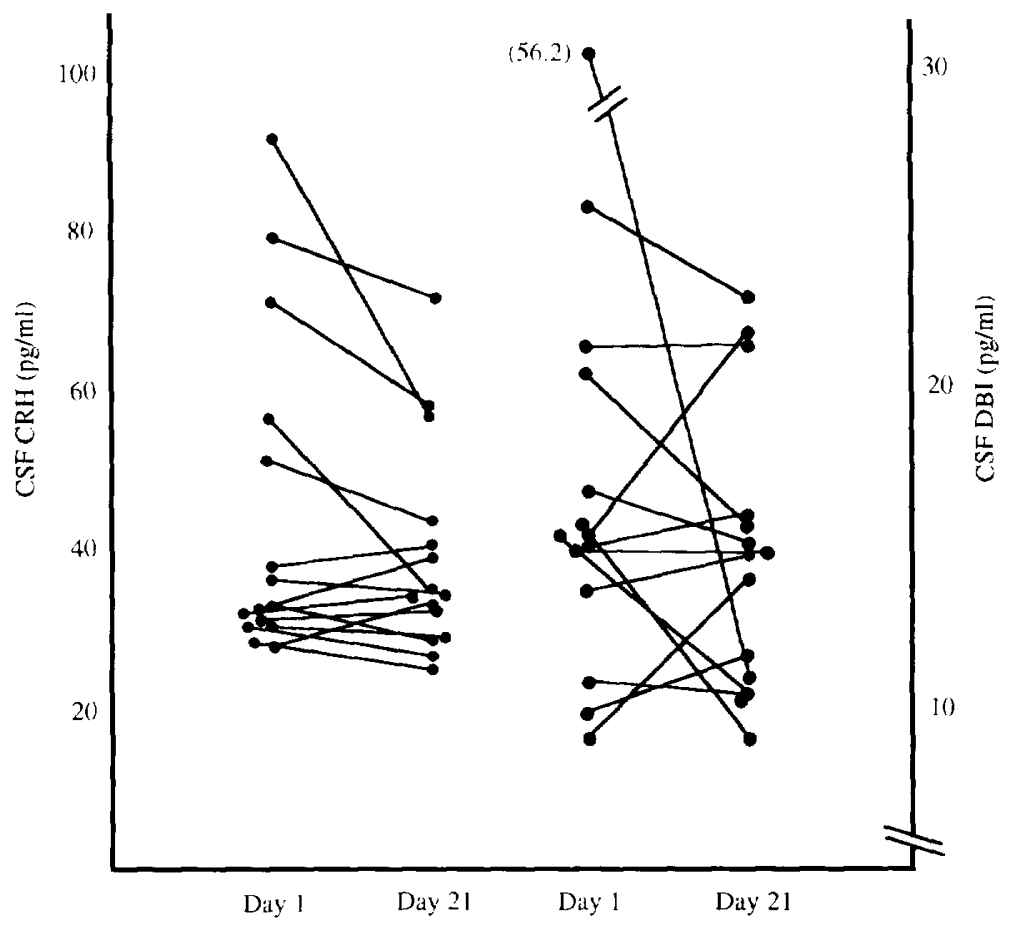

Figure 1. CSF CRH and DBI concentrations on Day 1 and Day 21 following the cessation of alcohol intake in alcohol-dependent patients. CSF CRH concentrations were higher on Day 1 than on Day 21 (paired one-tailed t-test, $p<.04$ ). CSF DBI concentrations were not significantly different between the two study days. 
Speilberger State $(r=-.63, \mathrm{df}=15, p=.01)$ and Hamilton Anxiety $(r=-.55, \mathrm{df}=15, p=.03)$. There was also no significant relationship between the change scores of the two neuropeptides. Demographic, clinical, and biological variables did not differentiate the six patients with decreasing CRH levels from the other patients.

\section{DISCUSSION}

CSF CRH concentrations were higher on Day 1 than Day 21. However, this difference was not as marked as those noted between plasma cortisol concentrations or the clinical variables. In addition, there were no significant correlations between CSF CRH concentrations during acute withdrawal and either plasma cortisol or clinical measures of withdrawal, and less than half of the study patients demonstrated CRH concentrations more than $12 \%$ higher on Day 1 than on Dav 21. Significant differences between CSF DBI concentrations were not evident in alcohol-dependent patients during acute alcohol withdrawal and at 3 weeks' absence, nor did concentrations of CSF DBI significantly correlate with measures of alcohol withdrawal severity. Limitations of the study design include the abstinence of a control group, the possibility of increased anxiety on Day 1 as a response to acute hospitalization rather than alcohol withdrawal, and the unknown test-retest reliability of both CRH and DBI.

CSF CRH concentrations reported here were within the range of $\mathrm{CRH}$ concentrations we and others have previously reported in healthy controls (Adinoff et al. 1990; Geracinto et al. 1994) and recently abstinent alcohol-dependent subjects (Adinoff et al. 1990). Geracinto et al. (1994), however, has recently reported that serially measures of CSF CRH in abstinent alcohol dependent patients were markedly lower than those of healthy controls. The CSF CRH concentrations in alcohol-dependent patients reported by Geracinto et al. were also much lower than those described here, possibly because of Geracinto et al.'s technique of placing the CSF catheter 3 hours before CSF sampling.

Our findings offer modest support for the hypothesis that central CRH release may be causally related to alcohol withdrawal symptoms, at least in some patients. The anxiogenic action of $\mathrm{CRH}$ has been indirectly implicated in the pathogenesis of alcohol withdrawal by both Baldwin et al. (1991) and Rassnick et al. (1993). Both groups have reported that the central administration of the CRH antagonist $\mathrm{CRH}_{4-41}$ reversed the anxiogeniclike effects of ethanol withdrawal. Furthermore, Pich et al. (1994) has recently reported that amygdalar CRH levels, as measured by microdialysis, were higher in rats following the cessation of an ethanol diet than in rats fed a control diet.
Although concentrations of CSF CRH were not consistently elevated in the present study, lumbar CSF may not provide an adequate measure of physiologically relevant brain regions. Using an indwelling subarachnoid catheter in patients and controls, Geracioti et al. (1992) measured CRH in lumbar CSF every 10 minutes for 6 hours. These investigators reported that $\mathrm{CRH}$ had a half-life of less than 10 minutes in lumbar CSF, suggesting that the spinal cord is the origin of lumbar CSF. Thus, measures of lumbar CSF CRH may be related as much to CSF flow dynamics as to the release of CRH from cortical regions.

The active disruption in a number of contributing neurophysiological systems (Adinoff 1994) may explain the absence of significant correlations between CSF $\mathrm{CRH}$ and clinical measures of withdrawal severity. The absence of a significant correlation between CSF CRH and plasma cortisol is consistent with several other reports indicating that CSF CRH concentrations are not significantly related to HPA axis functioning. Although Roy et al. (1987) noted a significant correlation between postdexamethasone cortisol and CSF CRH concentrations in depressed patients, this high correlation was not observed by Nemeroff et al. (1984) in a similar population. Significant correlations between CSF CRH and plasma cortisol have also not been reported in other control (Adinoff et al. 1990) and patient populations (Banki et al. 1987; Kaye et al. 1987; Nemeroff et al. 1984; Adinoff et al. 1990; Adinoff et al. 1991b; Geracioti et al. 1992, 1994). Furthermore, CSF CRH and plasma cortisol have been reported to exhibit opposing diurnal rhythms (Garrick et al. 1987; Kalin et al. 1987; Geracioti et al. 1992).

Our findings do not support the involvement of a $\mathrm{GABA}_{\mathrm{A}}-\mathrm{BZ}$ receptor-like endogenous ligand, such as $\mathrm{DBI}$, in the production of alcohol withdrawal symptoms. Sandler et al. (1984) has previously suggested that the endogenous substance "tribulin" may induce alcohol withdrawal through an interaction at the BZ receptor. However, the administration of the BZ antagonist flumazenil, which would be expected to antagonize the effects of such a compound, does not ameliorate withdrawal symptoms when administered during the acute phase of alcohol withdrawal (Little et al. 1985; Adinoff et al. 1986). If DBI were involved in the induction of withdrawal symptoms, an attenuated withdrawal response to flumazenil would have been expected. File et al. (1989) have reported that a single dose of flumazenil reversed withdrawal-related anxiety, although the extended response to this short-acting compound suggests that the mechanism of action did not involve the direct antagonism of an endogenous ligand.

The significant correlation between CSF DBI and CRH concentrations on Day $21(r=.71)$ is similar to the relationship between these two neuropeptides reported 
by Roy (1991) in healthy controls $(r=.55)$, depressed patients $(r=.68)$, and pathological gamblers $(r=.76)$. These findings may suggest that the concentrations of these compounds are influenced by similar neurobiological processes or that one of these neuropeptides controls the other's release. With the exclusion of one patient with a markedly elevated CSF DBI level on Day 1 , this relationship was also evident during acute withdrawal $(r=.75)$. Thus, the physiological relationship between $\mathrm{CRH}$ and $\mathrm{DBI}$ does not appear to be disturbed in alcohol-dependent subjects.

In a recently published report by Hawley et al. (1994), CSF concentrations of both CRH and DBI were measured in eight patients both during alcohol withdrawal and $11.9 \pm 8.1$ days after the first lumbar puncture. Hawley et al. found that neither neuropeptide significantly differed between both study days, confirming our findings with DBI but not CRH. However, there were several important differences between the study of Hawley et al. and the present study. First, in Hawley et al., the second lumbar puncture was not reported to be done at the same time of day as the first, complicating the interpretation because of potential circadian changes. Second, patients were chronically malnourished and required a lumbar puncture during alcohol withdrawal because of clinical indications (i.e., seizure, fever, or clinical suspicion of meningitis). These physiological disturbances could have influenced neuropeptide concentrations. Third, the timing (i.e., days after admission) of the second lumbar puncture varied considerably across patients, whereas the second lumbar puncture in the present study was consistently obtained on Day 21 of hospitalization. Finally, only eight patients were studied in the report by Hawley et al., increasing the possibility of a Type Il error.

The finding of increased CSF CRH in acutely withdrawing alcohol-dependent patients is not robust and clearly requires further study. The absence of any significant difference in CSF DBI concentrations between acute withdrawal and 3 weeks' abstinence time points may suggest either a relative stability of this neuropeptide in alcohol-dependent patients or that central changes in DBI concentrations are not reflected in lumbar CSF.

\section{ACKNOWLEDGMENTS}

This work was conducted at the Department of Psychiatry, Medical University of South Carolina, and the VA Medical Center, Charleston; Fidia-Georgetown Institute for the Neurosciences, Georgetown University School of Medicine; Department of Pharmacology, Duke University Medical Center. This work was supported by the Upjohn Company and NIMH grant MH-42088.

\section{REFERENCES}

Adinoff B, Majchrowicz E, Martin PR, Linnoila M (1986): The benzodiazepine antagonist $\mathrm{RO} 15-1788$ does not antagonize the ethanol withdrawal system. Biol Psychiatry 21:643-649

Adinoff B, Martin PR, Bone GHA, Eckardt MJ, Roehrich L, George DT, Moss H, Eskay R, Linnoila M, Gold PW (1990): Hypothalamic-pituitary-adrenal axis functioning and cerebrospinal fluid $\mathrm{CRH}$ and $\mathrm{ACTH}$ in alcoholics following recent and long term abstinence. Arch Gen Psychiatry 47:325-330

Adinoff B, Risher-Flowers D, De Jong J, Ravitz B, Bone GHA, Nutt DJ, Rochrich L, Martin PR, Linnoilla M (1991a): Disturbances of hypothalamic-pituitary-adrenal axis functioning during the ethanol withdrawal syndrome in six men. Am J Psychiatry 148:1023-1025

Adinoff B, Martin PR, Eckardt MJ, Bone GAH, Gold PW, Linnoila $M$ (1991b): Pituitary-adrenal responses to $\mathrm{CRH}$ and central neuropeptide levels in alcoholic amnestic disorder. Biol Psychiatry 29:1153-1155

Adinoff B, Linnoila M, Bissette G, Guidotti A, Mefford I, Nemeroff CB (1992): Cerebrospinal fluid concentrations of $\mathrm{CRH}, \mathrm{DBI}$, and catecholamine metabolites during alcohol withdrawal and abstinence. American College of Neuropsychopharmacology, San Juan, Puerto Rico, December 14

Baldwin HA, Rassnick S, Rivier J, Koob GF, Britton KT (1991): CRF antagonist reverses the "anxiogenic" response to ethanol withdrawal in the rat. Psychopharmacol 103:227-232

Banki CM, Bisette B, Arato $M, O^{\prime}$ Connor L, Nemeroff CB (1987): CSF Corticotropin-releasing factor-like immunoreactivity in depression and schizophrenia. Am J Psychiatry 144:873-877

Barbaccia ML, Costa E, Ferrero P, Guidotti A, Roy A, Sunderland T, Pickar D, Paul SM, Goodwin FK (1986): Diazepam binding inhibitor, a brain neuropeptide present in human spinal fluid: Studies in depression, schizophrenia, and Alzheimer's disease. Arch Gen Psychiatry 43:1143-1147

Bissette G, Reynolds GP, Kilts CD, Widerlov E, Nemeroff CB (1985): Corticotropin-releasing factor-like immunoreactivity in senile dementia of the Alzheimer type. JAMA 254:3067-3069

Breier A, Paul S (1990): The GABAN/benzodiazepine receptor: Implications for the molecular basis of anxiety. J Psychiatr Res 24:91-104

Britton KT, Lee G, Dana R, Risch SC, Koob GF (1986): Activating and "anxiogenic" effects of corticotropin releasing factor are not inhibited by blockade of the pituitaryadrenal system with dexamethasone. Life Sci 39:12811286

Costa E, Guidotti A (1991): Diazepam binding inhibitor (DBI): A peptide with multiple biological actions. Life Sci 49:325-344

Ferrarese C, Mennini T, Pecora N, Gobbi M, Appollonio I, Bernasconi P, Frigo M, Fegondi C, Pierpaoli C, Frattola L, Garattini S (1991): Acute noise stress in rats increases 
the levels of diazepam binding inhibitor (DBI) in hippocampus and adrenal gland. Psychopharmacology 103:339-342

Ferrero P, Santi MR, Conti-Tronconi B, Costa E, Guidotti A (1986): Study of an octadecaneuropeptide derived from diazepam binding inhibitor (DBI) biological activity and presence in rat brain. Proc Natl Acad Sci USA 83:827831

File SE, Baldwin HA, Hitchcott PK (1989): Flumazenil but nitrendipine reverses the increased anxiety during ethanol withdrawal in the rat. Psychopharmacology 98:262264

Garrick NA, Hill JL, Szele FG, Tomai TP, Gold PW, Murphy DL (1987): Corticotropin-releasing factor: A marked circadian rhythm in primate cerebrospinal fluid peaks in the evening and is inversely related to the cortisol circadian rhythm. Endocrinology 121:1329-1334

George DT, Zerby A, Noble S, Nutt DJ (1988): Panic attacks and alcohol withdrawal: Can subjects differentiate the symptoms? Biol Psychiatry 24:24()-243

Geracioti TD, Orth DX, Ekhator NN, Blumenkopf B, Loosen $P$ (1992): Serial cerebrospinal fluid corticotropin-releasing hormone concentrations in healthy and depressed humans. J Clin Endocrinol Metab 74:1325-1330

Geracioti TD, Loosen PT, Ebert MH, Ekhator NN, Burns ID, Nicholson WE, Orth DN (1994): Concentrations of corticotropin-releasing hormone, norepinephrine, MHPG, 5-hydroxyindoleacetic acid, and tryptophan in the cerebrospinal fluid of alcoholic patients: Serial sampling studies. Neuroendocrinology 60:635-642

Hawley RJ, Nemeroff CB, Bisette G, Guidotti A, Rawlings R, Linnoila M (1994): Neurochemical correlates of sympathetic activation during serere alcohol withdrawal. Alcohol Clin Exp Res 18:1312-1316

Kalin NH, Shelton SE, Barksdale CM, Brownfield MS (1987): A diurnal rhythm in cerebrospinal fluid corticotropinreleasing hormone different from the rhythm of pituitary-adrenal activity. Brain Res 426:335-341

Kaye WH, Gwirtsman HE, George DT, Ebert MH, Jimerson DC, Tomai TP, Chrousos Gl', Gold PW (1987): Elevated cerebrospinal fluid levels of immunoreactive corticotropin-releasing hormone in anorexia nervosa: Relation to state of nutrition, adrenal function, and intensity of depression. J Clin Endocrinol Metabol 64:203-208

Kling MA, Roy A, Doran AR, Calabrese JR, Rubinow DR, Whitfield HJ Jr, May C, Post RM, Chrousos GP, Gold PW (1991): Cerebrospinal fluid immunoreactive corticotropin-releasing hormone and adrenocorticotropin secretion in Cushing's disease and major depression: Potential clinical implications. J Clin Endocrinol Metab 72:260271

Kushner MG, Sher KI, Beitman BD (1990): The relation between alcohol problems and the anxiety disorders. Am J Psychiatry 147:685-695

Little HY, Taylor SC, Nutt DJ, Cowen PJ (1985): The benzodiazepine antagonist, Ro 15-1788, does not decrease ethanol withdrawal convulsions in rats. Eur J Pharmacol $107: 375-377$

Mendelson JH, Stein S (1966): Serum cortisol levels in alcoholic and nonalcoholic subjects during experimentally induced ethanol intoxication. Psychosom Med 28:616626

Millan MA, Jacobowitz DM, Hauger RL, Catt KJ, Aguiler G (1986): Distribution of corticotropin-releasing factor receptors in primate brain. Proc Natl Acad Sci USA 83: 1921-1925

Miyata M, Mocchetti I, Ferrarese C, Guidotti A, Costa E (1987): Protracted treatment with diazepam increases the turnover of putative endogenous ligands for the benzodiazepine/beta-carboline recognition site. Proc Natl Acad Sci USA 84:1444-1448

Morrow AL, Suzdak PD, Karanian JW, Paul SM (1988): Chronic ethanol administration alters $\gamma$-aminobutyric acid, pentobarbital and ethanol-mediated ${ }^{36} \mathrm{Cl}$-uptake in cerebral corticol synaptoneurosomes. J Pharmacology Experimental Ther 246:158-164

Nemeroff CB, Widerlov E, Bissette G, Walleus H, Karlsson K, Eklund K, Kilts CD, Loosen PT, Vale W (1984): Elevated concentrations of CSF corticotropin-releasing factor-like immunoreactivity in depressed patients. Science $126: 1342-1344$

Pich EM, Koob GF, Vale W, Weiss F (1994): Release of corticotropin-releasing factor (CRF) from the amygdala of ethanol-dependent rats measured with microdialysis. Alcohol Clin Exp Res 18:522

Rassnick S, Heinrichs SC, Britton KT, Koob GF (1993): Microinjection of a corticotropin-releasing antagonist into the central nucleus of the amygdala reserves anxiogeniclike effects of ethanol withdrawal. Brain Res 605:25-32

Rock JP, Oldfield EH, Schulte HM, Gold PW, Kornblith PL, Loriaux L, Chrousos GP (1984): Corticotropin releasing factor administered into the ventricular CSF stimulates the pituitary-adrenal axis. Brain Res 323:365-368

Roy A (1991): Cerebrospinal fluid diazepam binding inhibitor in depressed patients and normal controls. Neuropharmacology 30:1441-1444

Roy A, Pickar D, Paul S, Doran A, Chrousos GP, Gold PW (1987): CSF corticotropin-releasing hormone in depressed patients and normal control subjects. Am J Psychiatry 144:641-645

Roy A, DeJong J, Gold P, Rubinow D, Adinoff B, Ravitz B, Waxman R, Linnoila M (1990): Cerebrospinal fluid levels of somatostatin, corticotropin-releasing hormone and corticotropin in alcoholism. Acta Psychiat Scand $82: 44-48$

Sandler M, Glover V, Elsworth JD, Clow A (1984): Ethanol and endogenous ligands in humans. Psychopharmacol Bull 27:485-586

Stenzel-Poore MP, Heinrichs SC, Rivest S, Koob GF, Vale WW (1994): Overproduction of corticotropin-releasing factor in transgenic mice: A genetic model of anxiogenic behavior. J Neuroscience 14:2579-2584

Sudzak PE, Schwartz RD, Skolnick P, Paul SM (1986): Ethanol stimulates GABA receptor mediated chloride transport in rat brain synaptoneurosomes. Pro Natl Acad Sci U S A 83:4071-7075

Sullivan JT, Sykora K, Schneiderman J, Naranjo CA, Sellers EM (1989): Assessment of alcohol withdrawal: The 
revised clinical instrument assessment for alcohol scale (CIWA-AR). Br J Addictions 84:1353-1357

Sutton RE, Koob GF, LeMoal M, Rivier J, Vale W (1982): Corticotropin releasing factor produces behavioural activation in rats. Nature 297:331-333

Swiergiel AH, Takahashi LK, Kalin NH (1993): Attenuation of stress-induced behavior by antagonism of corticotropin-releasing factor receptors in the central amygdala in the rat. Brain Res 623:229-234

Valentino RJ, Foote SL (1986): Brain noradrenergic neurons, corticotropin-releasing factor, and stress. In Moody TW (ed), Neural and Endocrine Peptides and Receptors. New York, Plenum, pp. 101-119 